\title{
A Retrospective Study of Anesthetic Considerations in Hip Arthroscopy: Regional versus General
}

\author{
Molly Kraus $^{1 *}$, Brittany Foley${ }^{1}$, Lopa Misra1, David Hartigan², Marlene Girardo ${ }^{3}$, David Seamans ${ }^{1}$ \\ ${ }^{1}$ Department of Anesthesiology, Mayo Clinic, Phoenix, Arizona, USA \\ ${ }^{2}$ Department of Orthopedic Surgery, Mayo Clinic, Phoenix, Arizona, USA \\ ${ }^{3}$ Department of Health Sciences Research, Division of Biostatistics, Mayo Clinic, Phoenix, Arizona, USA \\ Email: *Kraus.molly@mayo.edu
}

How to cite this paper: Kraus, M., Foley, B., Misra, L., Hartigan, D., Girardo, M. and Seamans, D. (2018) A Retrospective Study of Anesthetic Considerations in Hip Arthroscopy: Regional versus General. Open Journal of Anesthesiology, 8, 295-304. https://doi.org/10.4236/ojanes.2018.812030

Received: November 30, 2018 Accepted: December 22, 2018 Published: December 25, 2018

Copyright $\odot 2018$ by authors and Scientific Research Publishing Inc. This work is licensed under the Creative Commons Attribution International License (CC BY 4.0).

http://creativecommons.org/licenses/by/4.0/

\begin{abstract}
Purpose: Hip arthroscopy is a surgery with favorable outcomes to treat labral tears of the hip. This retrospective review was conducted to identify an optimal anesthetic technique for hip arthroscopy to minimize postoperative pain and decrease opioid consumption. Methods: A retrospective analysis was performed for 92 patients who underwent hip arthroscopy for femoroacetabular impingement (FAI) with labral tear under general anesthesia $(G)$ with fascia-iliaca block (64 patients) or regional anesthesia (R) with fascia-iliaca block (28 patients) from March 9, 2016 to April 9, 2018. Data collected included: demographics, diagnosis, ASA status, time in surgery, medications administered, type of anesthesia administered, pain scores, use of straight catheter, and time in PACU. Results: Patients who underwent hip arthroscopy under regional anesthesia reported significantly less first (G: 3.4 (3.9), R: 1.3 (3.0), p = 0.0085) and average (G: 3.8 (2.5), R: $2.0(2.2), p=$ $0.0038)$ pain post-operatively. Patients under regional anesthesia also received less total morphine milligram equivalents (MME) intraoperatively and post-operatively (G: 40.8 (21.7) MME, R: 24.9 (17.8), $\mathrm{p}=0.004 \mathrm{MME)}$. Patients under regional anesthesia had a significantly higher incidence of urinary retention (G: 3.1\%, R: 28.6\%, p = 0.009) and increased time spent in PACU ( $G=181.9$ (86.3), R: 251.4 (80.4), $p=0.0001)$. Conclusions: The administration of regional anesthesia resulted in significantly lower pain scores and perioperative opioid consumption compared to general anesthesia in patients undergoing hip. This may be the optimal anesthetic technique for pain control; however, incidence of urinary retention and time to discharge are significantly increased. Prospective randomized control trials are needed to compare general anesthesia and regional anesthesia for hip arthroscopy.
\end{abstract}




\section{Keywords}

Anesthesia, Hip Arthroscopy, Perioperative Management, Regional, Spinal

\section{Introduction}

Hip arthroscopy is a growing field of orthopedics with dramatic increases in its incidence and indications [1] [2] [3]. Femoroacetabular impingement (FAI) with labral tear is one of the main indications for arthroscopy [4]. The surgery requires decompression of the offending bony anatomy with a burr (femoroplasty and acetabuloplasty) as well as repairing the injured labrum, which is the result of the two pathologic structures impinging on one another. This surgery is notable for significant improvement in patient reported outcomes, with high patient satisfaction [5] [6].

Despite the minimally invasive nature of arthroscopy, patients may experience notable pain post-procedure [7]. Contributing factors include complexity of hip innervation, individualized response to pain, as well as the surgical technique itself, which requires a large amount of distraction of the joint. Since these often are performed in an ambulatory setting, optimal control of pain and nausea is of utmost importance for safe and speedy discharge from the Post Anesthesia Care Unit (PACU). Over the last decade there have been several methods proposed to optimize pain control and decrease opioid consumption perioperatively.

Multiple studies have demonstrated perioperative nerve blocks are effective methods of providing adjunctive pain management. These include paravertebral, lumbar plexus, and fascia iliacus. However, there is no clear consensus on any one specific method that provides superior analgesia with reduced reported pain scores and adverse side effects [7]-[13]. To our knowledge, there are no studies comparing postoperative pain scores and opioid use between patients undergoing hip arthroscopies under general anesthesia versus those undergoing hip arthroscopies using regional anesthesia. We hypothesized that patients with spinal anesthetic for hip arthroscopy would have lower pain scores and less opioid consumption that those receive general anesthesia. This study is a retrospective review to compare the pain scores, opioid use and postoperative course in patients under general anesthesia compared to spinal anesthesia for hip arthroscopy.

\section{Methods}

Following IRB approval from Mayo Clinic (Rochester, MN), a retrospective analysis of data from patients who underwent hip arthroscopy procedures between March 9, 2016 and April 9, 2018 was performed. Inclusion criteria were adult patients undergoing hip arthroscopy during the time frame for hip impingement, femoroacetabular impingement (FAI), labral tear, or labral calcification by a single orthopedic surgeon. The following diagnoses were excluded 
from this study: abductor tear, gluteus medius/minimus tear, avascular necrosis, labral reconstruction, and IT band snapping. If any additional surgical technique was required besides hip arthroscopy, the case was excluded. Using an institutional data pull, 126 patients were identified as having undergone hip arthroscopy surgery by a single board-certified orthopedic surgeon at a single healthcare institution. Of the 126 patients, 92 met inclusion criteria and were included in this study. Thirty-four patients were excluded based on diagnoses or surgical technique as detailed below.

Abductor (gluteus medius/minimus) repairs and IT band snapping were excluded because they do not require traction and are performed on the extra-articular hip. Avascular necrosis was excluded because those cases were not completed entirely under arthroscopy. One patient was eliminated because he underwent a knee arthroscopy in addition to the hip arthroscopy.

Patients either received general anesthesia or spinal anesthesia for the procedure based on anesthesiologist, surgeon, and patient preference. A majority of patients in both groups also received a fascia iliacus block for postoperative pain control, the proportion receiving the block in both groups was similar and this was not statistically significantly different. Patients from both groups also received standard premedications for multimodal analgesia including acetaminophen, gabapentin, and celecoxib.

Utilizing an institutional chart review and data search, the following data was collected for analysis: demographics, preoperative and postoperative diagnosis, anesthesia type, preoperative, intraoperative, and postoperative pain medications, self-reported pain scores, PACU antiemetics administered, requirement of straight catheter, and time in PACU. Patients were asked to report their pain score using the Visual Analog Scale (VAS) on arrival to the PACU and every 15 minutes thereafter until discharge from the post anesthesia care unit. If patients receive opioid medication in PACU, they were asked to report their pain prior to receiving their next dose, which may vary from the standard 15 minute interval. First pain is the first recorded pain score in PACU. Minimum, maximum, and average pain scores are a measure of their overall reported pain scores in PACU. Steps were taken to prevent misclassification bias, the records were checked and verified based to anesthetic type classification, regional note recorded and review of the anesthetic record. To avoid recall bias, we used only information that was documented in real time in the chart.

\subsection{Statistical Analysis}

Descriptive statistics were used to summarize demographic information and to identify differences between the two groups. Chi-square test was used for categorical variables and Fisher's exact test, when appropriate. Mann-Whitney rank-sum test was used for continuous variables. Differences between general and regional anesthesia was determined by Pearson chi-square. P-values $<0.05$ were considered significant. Statistical analysis was performed using SAS version 9.4 (SAS Institute Inc.). 


\subsection{Anesthetic Techniques}

Fascia iliaca blocks were placed in the preoperative area with our standard procedure as follows; in the supine position, the inguinal crease was sterilized using chlorohexidine. After placing a 5 to $12 \mathrm{MHz}$ linear probe parallel to the inguinal ligament on the inguinal crease, the femoral artery, fascia lata, fascia iliaca, iliacus muscle, and femoral nerve were identified. Under ultrasound guidance, a 22 gauge (B Braun) 2-inch needle was inserted in-plane and advanced toward the fascia iliaca and iliacus muscle. After confirming the passage of the needle through the fascia iliaca, $30-40 \mathrm{ml}$ of either $0.5 \%$ ropivacaine, $0.25 \%$ bupivacaine, or $0.5 \%$ bupivacaine was injected in $5 \mathrm{ml}$ increments following frequent negative aspiration.

For patients who had general anesthesia, intravenous fentanyl, lidocaine, propofol, and rocuronium were typically given for induction and they were intubated in the normal fashion. Anesthesia was maintained with sevoflurane and additional pain medications were given at the discretion of the anesthesia team. For those patients in the regional group, awake patients were positioned either lateral decubitus or in the sitting position for spinal blocks, based on anesthesiologists' preference. All spinals were performed by experienced anesthesiologists at a single institution using standardized kits with sterile technique. Once cerebrospinal fluid was noted, $1.6 \mathrm{ml}-2 \mathrm{ml}$ of $0.75 \%$ preservative-free spinal bupivacaine was injected. Patients were also given sedation during the surgery, either midazolam, fentanyl or propofol infusion. All patients were monitored throughout the procedure using standard ASA monitoring.

\subsection{Surgical Technique}

Patients were all placed supine on an operative table with traction boot extensions. The anterolateral and mid-anterior portal were utilized in every patient. If suture anchors were required a distal lateral accessory portal was created. Diagnostic arthroscopy consisted of examination of ligamentum teres, labrum, and cartilage of the femoral head and acetabulum. Labral tears were repaired in all cases. Preoperative radiographs and intra-operative fluoroscopy were utilized for decisions whether bone needed to be resected for cam and/or pincer lesions. When resection was warranted fluoroscopy was utilized to ensure adequate resection was performed.

\section{Results}

Between March 9, 2016 and April 9, 2018, a total of 126 patients underwent a hip arthroscopy procedures performed by a single board-certified orthopedic surgeon, 92 of which are included in this study. In this analysis, 64 patients received General anesthesia $(G)$, and 28 patients received Regional spinal anesthesia (R). The ratio of men to women was 30:62 and the mean age was 34 years old. Average time in surgery was $108.5+/-31.5$ minutes. There is no statistical difference in patient demographics, length of surgery, or ASA physical status score 
between the two groups (Table 1 ).

Table 2 demonstrates, patients who received regional anesthesia reported significantly lower first pain (G: 3.4 (3.9), R: 1.3 (3.0), 0.0085), minimal pain (G: 1.7 (2.3), R: 0.4 (0.9), $\mathrm{p}=0.0027$ ), and average pain (G: 3.8 (2.5), R: 2.0 (2.2), $\mathrm{p}=$ 0.0038 ) scores postoperatively. (Capitalize Average pain score is equal to the sum of all pain scores reported in the PACU divided by the number of scores reported.) Reported maximum pain was also lower for patients in the regional group, however, this did not reach statistical significance (G: 5.6 (3.3), R: 4.1 (3.4), $p=0.0679$ ). A fascial iliaca block was administered to the majority of patients in both groups, resulting in no significant difference (G: $\mathrm{N}=55, \mathrm{R}: \mathrm{N}=24, \mathrm{p}=0.9774$ ) (Table 3). A greater percentage of patients who received general anesthesia were given pain medications in PACU, though not significant $(\mathrm{p}=0.1182)$. However, when combining the amount of opiates given intraoperatively and in PACU, patients who received general anesthesia received a significantly greater total amount of opiates (G: 40.8 (21.7) MME, R: 24.9 (17.8), $\mathrm{p}=0.0004 \mathrm{MME)}$ (Table 2).

There was no difference in the number of patients taking pain medication prior to the day of the procedure (G: $\mathrm{N}=6$ (9.4\%); $\mathrm{R}: \mathrm{N}=4(14.3 \%) ; \mathrm{p}=$ 0.2372). Due to the way the data was abstracted from the Electronic Medical Record (EMR), we are unable to determine whether these medications were prescribed at the patient's preoperative office visit or if the patients were consuming pain medication for other medical conditions prior to the procedure, however, there was no difference in the measure between groups. All patients were prescribed preoperative multimodal analgesics including celecoxib, acetaminophen, and gabapentin. Dosage adjustments were made according to age, renal function, and previous allergies or adverse events.

Spinal versus general anesthesia did not affect the number or dose of antiemetics administered to patients in the PACU. A higher percentage of patients in the general anesthesia group received antiemetics, but this figure did not reach statistical significance $(\mathrm{G}=45.3 \%, \mathrm{R}=28.6 \%, \mathrm{p}=0.1319)$.

Table 1. Demographics.

\begin{tabular}{ccccc}
\hline & General $(\mathrm{N}=64)$ & Regional $(\mathrm{N}=28)$ & Total $(\mathrm{N}=92)$ & P value \\
\hline Age at Surgery & & & & \\
Mean (SD) & $34.4(14.8)$ & $34.4(11.6)$ & $34.4(13.8)$ & \\
Range & $(14.0-77.0)$ & $(16.0-58.0)$ & $(14.0-77.0)$ & \\
Gender & & & \\
Female & $45(70.3 \%)$ & $17(60.7 \%)$ & $62(67.4 \%)$ & \\
Male & $19(29.7 \%)$ & $11(39.3 \%)$ & $30(32.6 \%)$ & \\
ASA & $38(59.4 \%)$ & $11(39.3 \%)$ & $49(53.3 \%)$ & \\
1 & $24(37.5 \%)$ & $15(53.6 \%)$ & $41(42.4 \%)$ & \\
2 & $2(3.1 \%)$ & $2(7.1 \%)$ & $4(4.3 \%)$ & \\
\hline
\end{tabular}


Table 2. Perioperative pain scores and MME.

\begin{tabular}{|c|c|c|c|c|}
\hline & $\begin{array}{l}\text { General } \\
(\mathrm{N}=64)\end{array}$ & $\begin{array}{l}\text { Regional } \\
(\mathrm{N}=28)\end{array}$ & $\begin{array}{c}\text { Total } \\
(\mathrm{N}=92)\end{array}$ & $\mathrm{p}$ value \\
\hline $\begin{array}{c}\text { Intraoperative MME } \\
\text { Mean (SD) }\end{array}$ & $22.7(8.1)$ & $11.4(7.6)$ & $19.2(9.5)$ & $<0.0001$ \\
\hline PACU MME Mean (SD) & $18.1(19.6)$ & $13.6(15.2)$ & $16.7(18.4)$ & 0.3025 \\
\hline Intra/PACU MME Mean (SD) & $40.8(21.7)$ & $24.9(17.8)$ & $36.0(21.8)$ & 0.0004 \\
\hline $\begin{array}{l}\text { Received Pain Medication in } \\
\text { PACU Mean (SD) }\end{array}$ & $43(67.2 \%)$ & $14(50.0 \%)$ & $57(62.0 \%)$ & 0.1182 \\
\hline First Pain & & & & 0.0085 \\
\hline Mean (SD) & $3.4(3.9)$ & $1.3(3.0)$ & $2.7(3.8)$ & \\
\hline Range & $(0.0-10.0)$ & $(0.0-10.0)$ & $(0.0-10.0)$ & \\
\hline Average Pain & & & & 0.0038 \\
\hline Mean (SD) & $3.8(2.5)$ & $2.0(2.2)$ & $3.2(2.6)$ & \\
\hline Range & $(0.0-9.5)$ & $(0.0-6.7)$ & $(0.0-9.5)$ & \\
\hline Min Pain & & & & 0.0027 \\
\hline Mean (SD) & $1.7(2.3)$ & $0.4(0.9)$ & $1.3(2.0)$ & \\
\hline Range & $(0.0-9.0)$ & $(0.0-3.0)$ & $(0.0-9.0)$ & \\
\hline Max Pain & & & & 0.0679 \\
\hline Mean (SD) & $5.6(3.3)$ & $4.1(3.4)$ & $5.1(3.4)$ & \\
\hline Range & $(0.0-10.0)$ & $(0.0-10.0)$ & $(0.0-10.0)$ & \\
\hline
\end{tabular}

Table 3. Perioperative measures.

\begin{tabular}{ccccc}
\hline & $\begin{array}{c}\text { General } \\
(\mathrm{N}=66)\end{array}$ & $\begin{array}{c}\text { Regional } \\
(\mathrm{N}=28)\end{array}$ & $\begin{array}{c}\text { Total } \\
(\mathrm{N}=94)\end{array}$ & P value \\
\hline $\begin{array}{c}\text { Preoperative Fascial } \\
\text { Iliacus Block }\end{array}$ & $55(85.9 \%)$ & $24(85.7 \%)$ & $79(85.9 \%)$ & 0.9774 \\
$\begin{array}{c}\text { Surgery Minutes } \\
\text { Mean (SD) }\end{array}$ & $106.1(29.2)$ & $114.0(36.2)$ & $108.5(31.5)$ & 0.5300 \\
Range & $(26.0-200.0)$ & $(78.0-223.0)$ & $(26.0-223.0)$ & 0.00021 \\
PACU Min & & & & \\
Mean (SD) & $184.0(87.4)$ & $251.4(80.4)$ & $204.1(90.4)$ & \\
Range & $(73.0-438.0)$ & $(83.0-397.0)$ & $(73.0-438.0)$ & \\
Straight Cath & & & & \\
within 24 hrs (y/n) & $2(3.1 \%)$ & $8(28.6 \%)$ & $10(10.9 \%)$ & 0.0009
\end{tabular}

As demonstrated in Table 3, on average, patients who received regional anesthesia spent $251.4+/-80.4$ minutes in PACU, which is significantly higher than patients who received general anesthesia (G: 181.9 (86.3), p =0.0001). A significantly higher percentage of patients in the regional anesthesia group received a straight catheter in PACU (G: 3.1\%, R: 28.6\%, p = 0.0009) (Table 2). 


\section{Discussion}

Despite the minimally invasive nature of arthroscopic surgery, hip arthroscopy can result in significant postoperative pain. Peripheral nerve blocks, such as fascia iliaca blocks, have been utilized in addition to multimodal analgesia to improve perioperative pain control in orthopedic surgeries, including arthroscopic hip surgery [7] [8] [11] [12] [13]. In this retrospective review we examined the pain management outcomes of patients who underwent hip arthroscopy under a general anesthetic compared to regional anesthesia plus sedation. The major findings were the reduced postoperative pain scores and the reduced opioid consumption in the regional group. Reported pain scores were significantly less for first pain, minimal pain, and average pain scores postoperatively. When comparing total opioid consumption, the regional group received significantly less opioids than the general group. These findings are consistent with earlier studies comparing regional and general anesthesia in regards topain, opioid use, and adverse complications in complex hip arthroscopies and open hip procedures [14].

In spite of the reduction in pain and opioid use, the regional group spent significantly more time in the recovery room prior to discharge and had higher incidence of urinary retention. Time to discharge is dependent on management of pain, nausea/vomiting, ability to urinate, and time for spinal block regression. We did not measure or report the regression of the spinal, however, the spinal was resolved prior to the patient's required physical therapy session which was completed before discharge. In our analysis, there was no difference between the groups in incidence of nausea and vomiting or antiemetic use in PACU, likely not contributing to the difference seen in time in PACU.

This was likely due to time needed for the regression of the spinal anesthetic and the higher incidence of urinary retention compared to the general group. The increased incidence of urinary retention for regional anesthesia in our analysis is consistent with prior study and is a contributing factor to the observed increased time to discharge [15]. These factors should be considered by the anesthesia care team when determining if improved pain management or decreased incidence of urinary retention and earlier discharge is of higher consideration for the individual patient.

The decrease in pain and opioid consumption is encouraging but it would be more notable if this reduction continues after discharge. Studies on surgical patients show that patients are at an increased risk of chronic opioid consumption with each additional week of use postoperatively [16] [17] [18] [19]. In a study of over 1 million post-surgical patients, $0.6 \%$ were consuming opioids one month postoperatively, and $0.2 \% 1$ year postoperatively [18]. Orthopedic surgeries were the most common type of surgery leading to prolonged opioid use in this study. Prolonged use has an increased risk of opioid dependence, pathologic pain, as well as multisystem organ dysfunction [18]. Unfortunately, in our study we were unable to assess pain and opioid use in the post-operative period after discharge. 
Further prospective studies in this population could assess whether adequate pain management with regional anesthesia and adjuvant peripheral nerve block can reduce the patients' overall opioid consumption in quantity and time course and therefore, reducing the risk for using opioids long term.

\section{Limitations}

This analysis is based on retrospective data in which patients were not randomized and the choice of anesthesia was based on surgeon, anesthesiologist, and patient preference. These factors potentially bias our results. Additionally, a patient may have received a higher amount of opioids intraoperatively or immediately postoperatively due to biased expectations of intraoperative and postoperative providers. Further, we did not control for patients who had a previous diagnosis of chronic pain syndrome or a history of narcotic use prior to surgery, although preoperative narcotic use was not significantly different between the two groups. The lack of standardization in recording pain scores at specific time points could create confounding variability in pain scores. Additionally, our analysis did not include data on pain or opioid use after discharge from the hospital, so we can only conclude that regional anesthesia lowered pain scores and intraoperative and immediate postoperative opioid consumption.

There was no standard protocol for preventing urinary retention in patients receiving spinal anesthetic at the time this data was collected. Implementing a protocol that includes limiting fluids, having patients empty their bladder prior to surgery and perhaps straight catheterization intraoperatively may reduce the incidence of straight catheterization in the PACU. All spinals in this study were with bupivacaine and the findings may not be generalizable to spinals using other local anesthetics. The time between performance of the fascia iliaca block and the induction of anesthesia did not allow for accurate assessment of the effectiveness of the blocks. The assumption that there would be an equivalent percentage of successes and failures in each group may be inaccurate. A randomized, prospective study on this subject is needed to eliminate these possible biases.

\section{Conclusion}

For hip arthroscopy, administration of a regional anesthetic combined with multimodal analgesics and a peripheral nerve block significantly reduced postoperative pain scores in PACU and reduced the perioperative use of opioids compared to general anesthesia combined with multimodal analgesics and a peripheral nerve block. However, this technique may result in urinary retention and a longer recovery time in PACU. Further clinical studies are needed to clearly verify the superior efficacy of pain management with a lower side-effect profile in comparison to general anesthesia.

\section{Conflicts of Interest}

The authors declare no conflicts of interest regarding the publication of this paper. 


\section{References}

[1] Botser, I.B., Smith, T.W., Nasser, R. and Domb, B.G. (2011) Open Surgical Dislocation versus Arthroscopy for Femoroacetabular Impingement: A Comparison of Clinical Outcomes. Arthroscopy. The Journal of Arthroscopic \& Related Surgery, 27, 270-278. https://doi.org/10.1016/j.arthro.2010.11.008

[2] Bozic, K.J., Chan, V., Valone, F.H., Feeley, B.T. and Vail, T.P. (2013) Trends in Hip Arthroscopy Utilization in the United States. Journal of Arthroplasty, 28, 140-143. https://doi.org/10.1016/j.arth.2013.02.039

[3] Maradit Kremers, H., Schilz, S.R., Van Houten, H.K., et al. (2017) Trends in Utilization and Outcomes of Hip Arthroscopy in the United States between 2005 and 2013. Journal of Arthroplasty, 32, 750-755. https://doi.org/10.1016/j.arth.2016.09.004

[4] Nepple, J.J., Prather, H., Trousdale, R.T., et al. (2013) Clinical Diagnosis of Femoroacetabular Impingement. Journal of the American Academy of Orthopaedic Surgeons, 21, S16-S19.

[5] Jamil, M., Dandachli, W., Noordin, S. and Witt, J. (2017) Hip Arthroscopy: Indications, Outcomes and Complications. International Journal of Surgery, 54,341-344. https://doi.org/10.1016/j.ijsu.2017.08.557

[6] Ross, J.R., Larson, C.M. and Bedi, A. (2017) Indications for Hip Arthroscopy. Sports Health, 9, 402-413. https://doi.org/10.1177/1941738117712675

[7] Kay, J., De Sa, D., Memon, M., Simunovic, N., Paul, J. and Ayeni, O.R. (2016) Examining the Role of Perioperative Nerve Blocks in Hip Arthroscopy: A Systematic Review. Arthroscopy. The Journal of Arthroscopic \& Related Surgery, 32, 704-715. https://doi.org/10.1016/j.arthro.2015.12.022

[8] Krych, A.J., Baran, S., Kuzma, S.A., Smith, H.M., Johnson, R.L. and Levy, B.A. (2014) Utility of Multimodal Analgesia with Fascia Iliaca Blockade for Acute Pain Management Following Hip Arthroscopy. Knee Surgery, Sports Traumatology, Arthroscopy, 22, 843-847. https://doi.org/10.1007/s00167-013-2665-y

[9] Garner, M., Alsheemeri, Z., Sardesai, A. and Khanduja, V. (2017) A Prospective Randomized Controlled Trial Comparing the Efficacy of Fascia Iliaca Compartment Block versus Local Anesthetic Infiltration after Hip Arthroscopic Surgery. Arthroscopy: The Journal of Arthroscopic \& Related Surgery, 33, 125-132. https://doi.org/10.1016/j.arthro.2016.10.010

[10] Childs, S., Pyne, S., Nandra, K., Bakhsh, W., Mustafa, S.A. and Giordano, B.D. (2017) The Effect of Intra-Articular Cocktail Versus Femoral Nerve Block for Patients Undergoing Hip Arthroscopy. Arthroscopy. The Journal of Arthroscopic \& Related Surgery, 33, 2170-2176. https://doi.org/10.1016/j.arthro.2017.06.036

[11] Dold, A.P., Murnaghan, L., Xing, J., Abdallah, F.W., Brull, R. and Whelan, D.B. (2014) Preoperative Femoral Nerve Block in Hip Arthroscopic Surgery: A Retrospective Review of 108 Consecutive Cases. American Journal of Sports Medicine, 42, 144-149. https://doi.org/10.1177/0363546513510392

[12] Ward, J.P., Albert, D.B., Altman, R., Goldstein, R.Y., Cuff, G. and Youm, T. (2012) Are Femoral Nerve Blocks Effective for Early Postoperative Pain Management after Hip Arthroscopy? Arthroscopy: The Journal of Arthroscopic \& Related Surgery, 28, 1064-1069. https://doi.org/10.1016/j.arthro.2012.01.003

[13] Xing, J.G., Abdallah, F.W., Brull, R., et al. (2015) Preoperative Femoral Nerve Block for Hip Arthroscopy. American Journal of Sports Medicine, 3. https://doi.org/10.1177/0363546515602468

[14] Indelli, P.F., Grant, S.A., Nielsen, K. and Vail, T.P. (2005) Regional Anesthesia in 
Hip Surgery. Clinical Orthopaedics and Related Research, 441, 250-255. https://doi.org/10.1097/01.blo.0000192355.71966.8e

[15] Mulroy, M.F., Larkin, K.L., Hodgson, P.S., Helman, J.D., Pollock, J.E. and Liu, S.S. (2000) A Comparison of Spinal, Epidural, and General Anesthesia for Outpatient Knee Arthroscopy. Anesthesia \& Analgesia, 91, 860-864. https://doi.org/10.1097/00000539-200010000-00017

[16] Brummett, C.M., Waljee, J.F., Goesling, J., et al. (2017) New Persistent Opioid Use after Minor and Major Surgical Procedures in US Adults. JAMA Surgery, 152, e170504. https://doi.org/10.1001/jamasurg.2017.0504

[17] Alam, A., Gomes, T., Zheng, H., Mamdani, M.M., Juurlink, D.N. and Bell, C.M. (2012) Long-Term Analgesic Use After Low-Risk Surgery A Retrospective Cohort Study. Archives of Internal Medicine, 172, 425-430. https://doi.org/10.1001/archinternmed.2011.1827

[18] Brat, G.A., Agniel, D., Beam, A., et al. (2018) Postsurgical Prescriptions for Opioid Naive Patients and Association with Overdose and Misuse: Retrospective Cohort Study. BMJ, 360, j5790. https://doi.org/10.1136/bmj.j5790

[19] Clarke, H., Soneji, N., Ko, D.T., Yun, L. and Wijeysundera, D.N. (2014) Rates and Risk Factors for Prolonged Opioid Use after Major Surgery: Population Based Cohort Study. BMJ, 348, g1251. https://doi.org/10.1136/bmj.g1251 